\title{
Operation Indices for Smart Power Dispatch Center Design
}

\author{
Jianing Liu ${ }^{1}$, Puming Li $^{1}$, Jin Zhong ${ }^{2}$, Liang Liang ${ }^{2}$ \\ ${ }^{1}$ Power Dispatch Center, Guangdong Power Grid Co., Guangzhou, China \\ ${ }^{2}$ Department of Electrical \& Electronic Engineering, The University of Hong Kong, Hong Kong, China \\ Email: jzhong@eee.hku.hk
}

Received 2013

\begin{abstract}
With the development of power systems, power grid within a control area becomes much more complicated due to increasing number of nodes and renewable energy interconnections. The role of power system control center is more critical in maintaining system reliable and security operations. Latest developed information and communication technologies provide a platform to enhance the functions and performance of power system control center. Smart power dispatch concept will be the trend of future control center development. In this paper, we start from the human factors of control center design and propose operation indices to reduce the information presented to the system operator. The operation indices will be the important criteria in situation awareness of a power grid. Past, present, future and capability states of a power grid are also proposed to provide better visions to the operator of system conditions. The basic ideas of operation indices and operation states are discussed in the paper. In the end, the design factors for a power dispatch cockpit are discussed.
\end{abstract}

Keywords: Control Center; Power Dispatch; Operation Index; Power System Operation; Human Factors; Power Dispatch Cockpit.

\section{Introduction}

Control center is the soul of a power system for its operations. The task of control center is to maintain real-time balancing of power supply and demand while achieve the security, reliability and economic requirements of power system operation. The concept of smart grid was raised after the 2003 North American blackout. The purpose of smart grid is to upgrade existing power systems with latest communication, power electronics and control technologies [1]. Besides the upgrading of power generation, transmission and distribution facilities, a smart control center using latest technologies is essential for future smart grid. The development of control center used to follow the requirement of power system operation and management, while updated with the development of communication technologies. In a smart grid, the functions of a control center and the way of power dispatch will have an essential change to adapt newly developed smart grid.

The development of supervisory control and data acquisition (SCADA) system could be traced back to the 1965 blackout of New York [2]. Power system measurement and monitoring system was developed. Later, advanced applications like security analysis, load forecasting, generation scheduling, and contingency analysis were developed. With the monitoring data from SCADA and the advanced applications, energy management system (EMS) was deployed. The SCADA/EMS system has been used in almost all power control centers in the world for power system monitoring, analysis and control. The basic concepts and framework of SCADA/EMS were deployed in 1970s-1980s [3]. The upgrading in recent years is mainly focus on software implementation, visualization and communication [4-6]. Much more data and information is available to the system operator with the upgrading of communication and data acquisition methods. With the latest generation of SCADA/EMS, the system operator is facing a huge amount of data, both real-time and offline data. The data available to the system operator have been increased exponentially. However, the design concept of EMS still keep the same as before, the system operator monitors operation data and needs to make operation decisions in the emergent situation according to their experiences or base on the calculation results of off-line decision tools. The design framework works well in the past, as the amount of data 
available to the operator is limited. Nowadays, plenty of data acquired from SCADA system is available to the system operator. In contingency situations, it is hard for the system operator to quickly locate useful information, analyze power flow distribution, re-allocate power flow and modify network topology. Although latest EMS systems have better analytical abilities, manual operations from system operators are still necessary. To safely operate a complicated system as large-scale power system, human factors and visualizations techniques need to be considered in the design of control center to improve the performance and decision making by the system operator in emergent situations.

In this paper, we will discuss the philosophy of control center design considering human factors and ergonomics. To simplify the data faced to the system operator, we will propose several important operation indices for the system operator to make quick decisions. In the end, the concept of dispatch cockpit for a power system control center will be presented.

\section{Control Center Design Issues}

\subsection{Man-Machine Model}

Power system is the most complicated system in the world not only because of its large amount of bus-bars, generation and transmission devices. In different operation states, voltage and power parameters are different, and they indicate the trend of future states. Automatic breaker actions due to fault events or overloading change the topology of the network. The large number of devices and the real-time changing of operation states produce a lot of real-time information to the system operator, whose work is to receive information and act on the basis of the information to control the system. This is a man-machine model.

A well designed interface and framework of a man-machine model could significantly improve the efficiency of the control system. Especially during the emergency states, quick and correct actions to the events are very important. The issue in power system control center is that the system operator needs to handle too much information in very short time to react to emergency situation. A clear indication of operation states and simplified (after analysis) information could release the pressure to the system operator and help them to make quick decisions.

\subsection{Visualization and Human Factors in Control Centers}

The man-machine interaction has been applied in the de- sign of EMS and power system control center. For example, one-line diagram, 2-D visualization and geographic information are used to display the power grid. Multiple screens are used for displaying of grid topology in multiple layers. 3-D visualization and animation are also proposed for power system analysis [7], which could easily indicate severe voltage problem or over-loading. Visualization software is also developed to display power flow and operation data in a user-friendly interface [8]. A Web-based tool with flexible open data structure is proposed to serve as a platform for power system visualization [9]. In 1980s, human factors have been considered in the design of dispatch control centers for utilities to overcome stressful situations in system operations [10]. The major states of power system operations are normal, degraded, and restorative. Therefore, the operator's activities can be categorized as pre-dispatch, dispatch and post-dispatch. Raw data needs to be converted to useful necessary information for three different time lines.

\section{Operation Indices for Different Operation States}

\subsection{Operation States for Power System Dispatch}

In traditional power system dispatch and state estimation, system operations states are classified as normal, degraded and restorative states. With our operation experiences of a large-scale power grid, fours states with simple indices are proposed. The four states are past state, present state, future state and capability state. For present state, it shows current situation of the system, for example, whether it is normal state, abnormal state or in emergent. The present state will become past state with time passing. The future state is calculated based on the system forecasting result, current system parameters and raw data. Analytical tools need to be used to calculate the state parameters of present and future states. Capability state shows the stability capability of a system. It is somewhere between present state and future state. The definition and calculation of the state are based on the system reserve, loading margin, etc. The four states and its times lines are shown in Figure 1. For present and future states, each of them has normal, abnormal and emergent states. The relationship and interaction between the normal, abnormal and emergent states are show as in Figure 2.

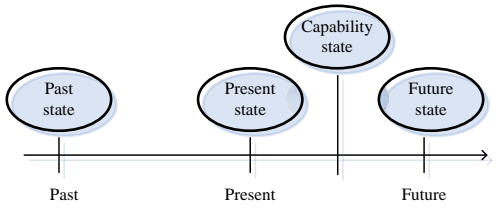

Figure 1. Four states with timelines 


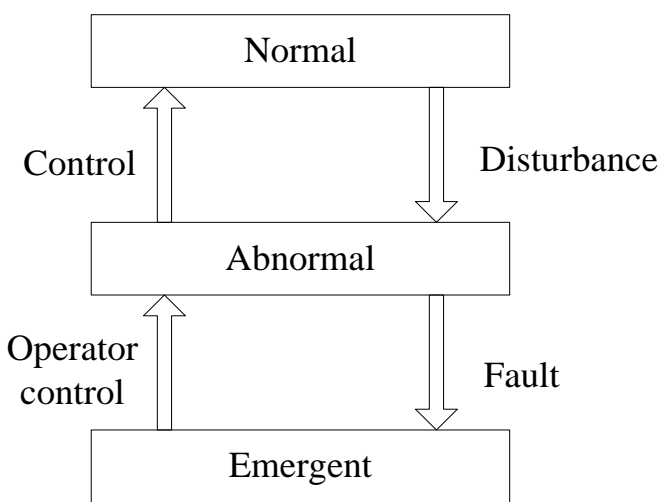

Figure 2. Normal, abnormal and emergent states and their control relationship

\subsection{Key Performance Indicator}

In the complicated industry control system and management cockpit, key performance indicator (KPI) is used as the useful information converted from raw data to simply tell the situation of the system. The KPIs can be used as the benchmark to sense the operation situations, hence to achieve situation awareness. In this paper, we will propose an index system for power system dispatch from different time scales and various aspects: security, economic, environmental and operation risk. The compromised index considering four indices can evaluate the operation state of the grid. The indices are shown as in Figure 3.

\section{Security Index}

Security is the most important issue in power system operation. Security index plays an important role in deciding system operation states and operational risks. The security index mainly affects present state monitoring and future state monitoring. Figure 4 shows the position and relationship of a security index with other operation states and data. Figure 5 shows the definition of security index.
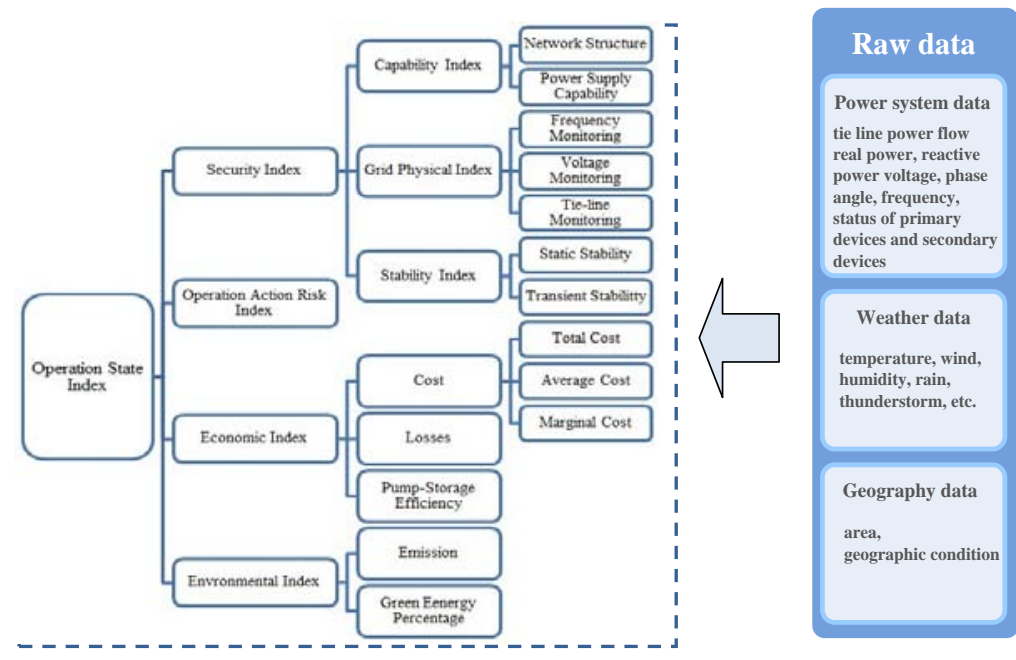

Figure 3. Structure of operation indices

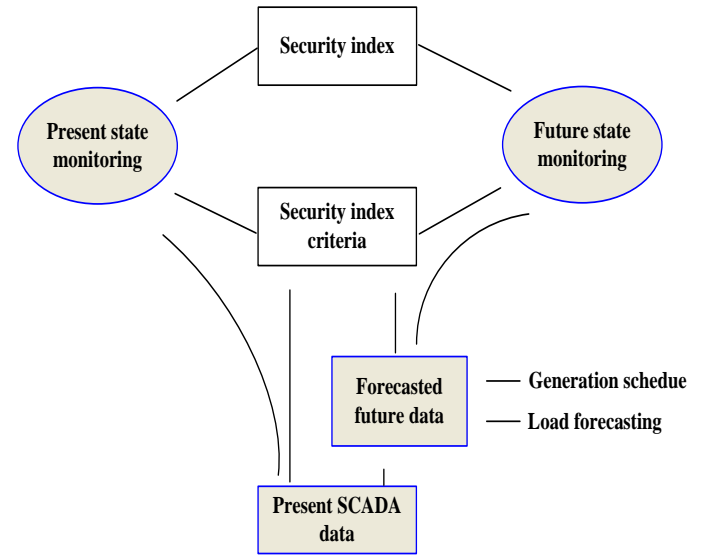

Figure 4. Security index with operation states

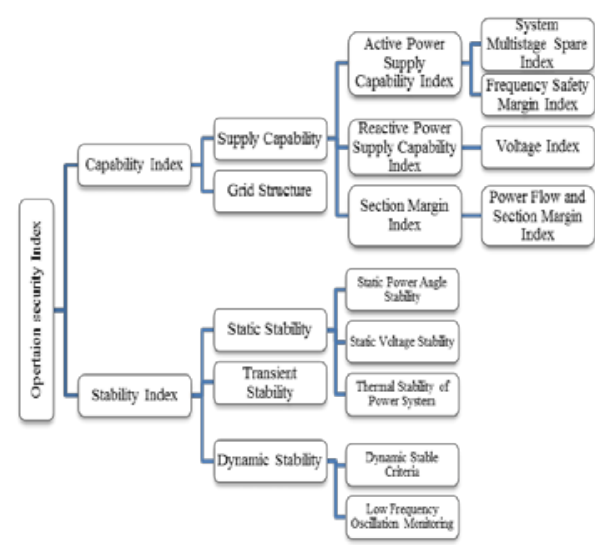

Figure 5. Security index. 


\section{Economic Index}

Economic factor is one of the concerns in power system dispatch. In Guangdong power grid, pump-storage capacity is high. It is used for quick reserve and energy storage. The economic benefits of pump-storage need to be considered when dispatching pump-storage stations. The other factors need to be considered are total cost for power generation and purchasing, electricity average price and cost of transmission losses. The definition of economic index is shown in Figure 6.

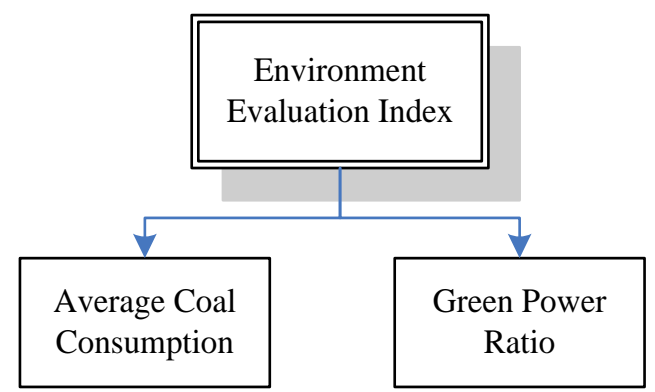

Figure 6. Economic index

\section{Environmental Index}

Carbon emission and other environmental issues need to be considered in power system dispatch nowadays due to the policy of renewable energy target and emission control. The environmental index can be simply considered based on the emission of coal-fired power plants and the penetration of renewable energy generation in a grid, as shown in Figure 7.

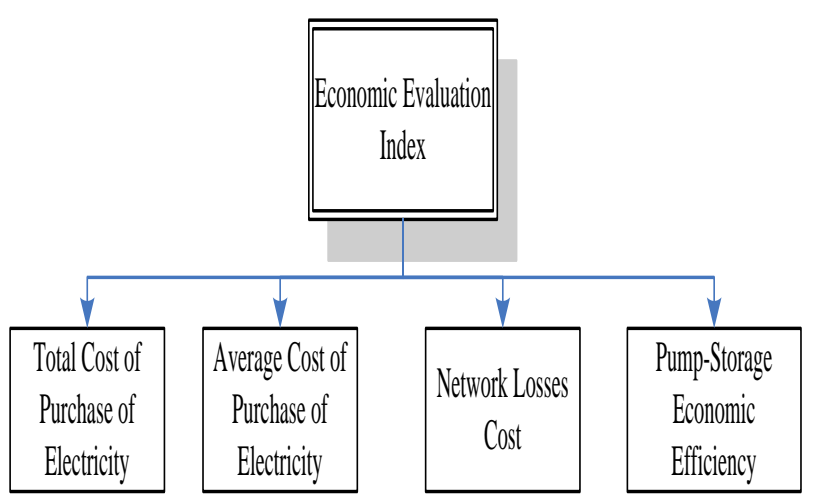

Figure 7. Environmental index

\subsection{Applying KPIs to Operation States}

The security index, economic index and environmental index can be calculated for present state, future state and past state based on the raw data from SCADA and analytical results of advanced applications. The normal, abnormal and emergent status can be easily displayed for past, present and future operation. It simplifies the information to the system operator.

Capability state is calculated in a separate way. It shows the capability of a system under the situation that all security criteria are satisfied. The index of capability state should reflect real-power adjustment capability, network topology, transmission margin, system reserve, frequency margin, etc., as shown in Figure 8.

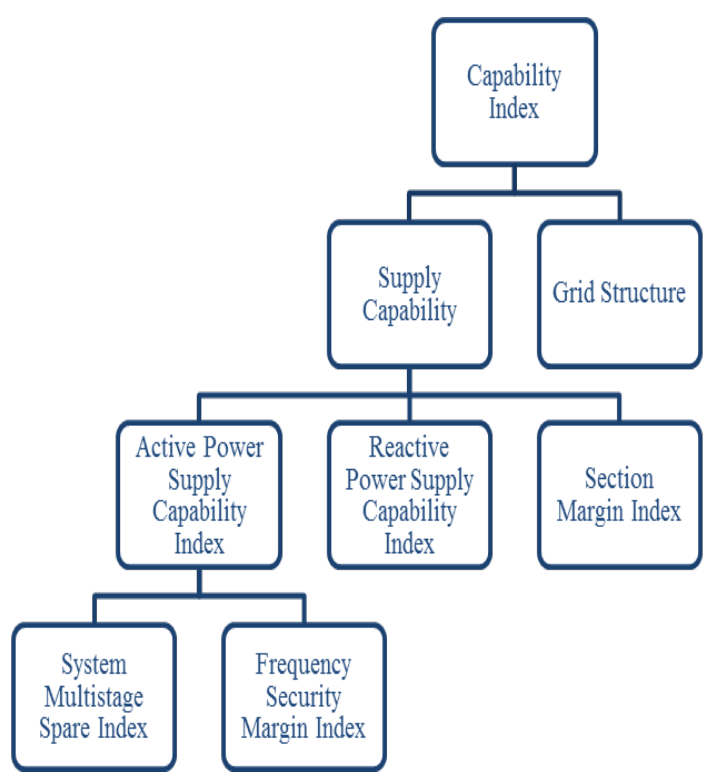

Figure 8. Capability state and its indices

\section{Design of Cockpit for Power System Con- trol Center}

Dispatching and operating a power system is more or less like controlling a complicated system or driving an aircraft to be sure that everything is on the right way. The control objective of power control center is to maintain nominal frequency and also be sure all facilities are operated under normal situation. If there a fault event, quick actions are needed to release the fault as well as minimize the number of customers affected / blackout area.

In China Southern Grid (CSG), the concept of dispatch cockpit has been proposed for control center design. The concept base on the fact that the man machine interaction in power control center is similar as the man machine control in the cockpits of aircraft or other industrial control systems. The power system operator needs to aware the situation by eyes, ears and brain, and control the system by hand. The operational decisions made according to real-time information aim to operate the system as pre-scheduled, as well as within security regions. Key 
performance indicators designed for power dispatch center condense information from raw data; provide visible information to indicate the system situation.

The KPIs of present state and future state present most important information to the system operator. If both of them are in normal states (with green light), the system operator could relax. Once alert states (for example yellow light) appear, the system operator needs to quickly check the system data and trace back to find the source of the emergency, and remove the risk through certain operations. If the system shows that the future state is abnormal (red light), the system operator needs to quickly adjust the system parameters to control the future state to be back to normal state. If the index of capability state shows that the system operation margin is not that good, the system operator needs to adjust the system conditions to guarantee enough security margin and reserves. KPI design and the accuracy of its calculation are very important in this case.

The other concept introduced to the power dispatch cockpit is the functions required by different management levels. For example, the system operators need all monitoring and control functions in the control center. The upper level managers could have smaller cockpits in their offices. Only information related to their management jobs is displayed in their office cockpits. Different departments have different access rights to operation information, and different authorizations on controlling the system.

Human factors and ergonomics design have great impacts on operator's reaction speed to emergent events. A well-designed cockpit system could also reduce the pressure of the system operator during normal situation. To design a good cockpit, practical experiences of system operators are extremely important. The human factors design for a power dispatch center could from four aspects:

- Monitoring: flexible monitoring layers are needed for monitoring power flow interfaces. It would be better to have flexible-defined power flow interfaces and limits, which will help in deciding alerting states for present and future situations.

- Control: real power adjustment and control methods are essential. Friendly man machine control design will increase the control accuracy.

- Decision making: automatic calculation and analysis based on monitoring data is needed. Power flow interface parameter calculations and adjustment strategy notifications are important if they could be implemented automatically and timely.

- $\quad$ Alert: the way of alert could affect the system operator. A good alerting system could reduce system operator's pressure in normal situation as well as improve their responding speed to emergent events.

- Latest information technologies could improve system operators control on the system. For example, most people use pad and smart phones nowadays. The applications of touch screens, pad computers and other mobile devices in control center could improve the work efficiency.

\section{Conclusions}

The paper discussed the issues of control center design from human factors aspects. The operation indices of security, economic and environmental are proposed to reduce the amount of information faced by the system operator. The operation indices are applied to past, present, future, and capability states to simply indicate system operation conditions. All these designs could be applied in the control center to improve the efficiency of man machine control. The new concepts of power dispatch cockpits are also presented and discussed in the paper.

\section{Acknowledgements}

The work is sponsored by the Dispatch Center of Guangdong Power Grid Co., Guangzhou, Guangdong, China. The project number is K-GD2012-300.

\section{REFERENCES}

[1] H. Farhangi, "The Path of the Smart Grid," IEEE Power \& Energy Magazine, January/February 2010, pp. 18-28.

[2] U.S. Federal Energy Commission, "Final report on 1965 blackout,” July 19, 1967.

[3] Thomas Kropp, "System Threats and Vulnerabilities: an EMS and SCADA Security System Overview," IEEE Power \& Energy Magazine, March/April 2006, pp.46-50.

[4] C. Zheng, "Data Integration Used in New Applications and Control Center Visualization Tools," IEEE Power and Energy Society General Meeting, July 2010.

[5] S.P. Carullo, "Interconnected Power Systems Laboratory: a Computer Automated Instructional Facility for Power System Experiments," IEEE Transactions on Power Systems, Vol. 17, pp. 215-222, 2002.

[6] A. Mercurio, A. Di Giorgio and P. Cioci. "Open-Source Implementation of Monitoring and Controlling Services for EMS/SCADA Systems by Means of Web ServicesIEC 61850 and IEC 61970 Standards," IEEE Transactions on Power Delivery, Vol. 24, pp. 1148-1153, 2009.

[7] F. Milano, "Three-Dimensional Visualization and Animation for Power Systems Analysis," Electric Power Systems Research Vol. 79, 2009, pp. 1638-1647. doi:10.1016/j.epsr.2009.06.009

[8] T. J. Overbye, and J. D. Weber, "Visualizing the Electric Grid,” IEEE Spectrum, Feb. 2011, pp. 52-58. 
[9] F. Li, "Web Tool Opens Up Power System Visualization,” IEEE Power \& Energy Magazine, July/August 2003, pp. 37-41.

[10] C. J. Frank, R. A. Bednarik, K. N. Zadeh, H. D. McNair, and C. J. Goddard, "Dispatch Control Center Human
Factors - Revisited," IEEE Transactions on Power Apparatus and Systems, Vol. PAS-104, No. 6, June 1985, pp. 1294-1299. 